\title{
Carbon dioxide-pneumoperitoneum in rats reduces ischemia/reperfusion-induced hepatic apoptosis and inflammatory responses by stimulating sensory neurons
}

\author{
MIHO KAWAI $^{1}$, JUAN ZHAO ${ }^{2}$, HIDEYUKI ISHIGURO ${ }^{1}$ and HIROMITSU TAKEYAMA ${ }^{1}$ \\ Departments of ${ }^{1}$ Gastroenterological Surgery and ${ }^{2}$ Translational Medical Science Research, Nagoya City University, \\ Graduate School of Medical Sciences, Nagoya, Aichi 467-8601, Japan
}

Received August 6, 2013; Accepted April 16, 2014

DOI: $10.3892 / \mathrm{mmr} .2014 .2329$

\begin{abstract}
Laparoscopic surgery induces a milder inflammatory response than open surgery, however, the precise mechanisms underlying this phenomenon remain to be elucidated. Our previous study demonstrated that stimulation of sensory neurons inhibited hepatic apoptosis and inflammatory responses in rats subjected to hepatic ischemia/reperfusion (I/R). Since carbon dioxide $\left(\mathrm{CO}_{2}\right)$ has been demonstrated to stimulate sensory neurons, it was hypothesized that $\mathrm{CO}_{2}$-pneumoperitoneum, as used in laparoscopic surgery, may attenuate inflammatory responses by stimulating sensory neurons. This hypothesis was examined using rats subjected to hepatic I/R. The rats were subjected to partial hepatic ischemia for 60 min followed by reperfusion. Abdominal insufflation with $\mathrm{CO}_{2}$ or air was performed for $30 \mathrm{~min}$ prior to hepatic I/R. Hepatic I/R-induced hepatocellular apoptosis and expression of the neutrophil chemoattractant endothelial monocyte-activated polypeptide-II, were inhibited by $\mathrm{CO}_{2}$-pneumoperitoneum, however, not by air-pneumoperitoneum. Pretreatment with the transient receptor potential vanilloid 1 antagonist SB366791 reversed the protective effects of $\mathrm{CO}_{2}$-pneumoperitoneum. The results from the present study demonstrated that $\mathrm{CO}_{2}$-pneumoperitoneum attenuates hepatic apoptosis and inflammatory responses in rats subjected to hepatic I/R, possibly by stimulating sensory neurons. These findings suggested that $\mathrm{CO}_{2}$-pneumoperitoneum contributed to the attenuated inflammatory response observed following laparoscopic surgery.
\end{abstract}

Correspondence to: Professor Hiromitsu Takeyama, Department of Gastroenterological Surgery, Nagoya City University, Graduate School of Medical Sciences, Kawasumi 1, Mizuho-cho, Mizuho-ku, Nagoya, Aichi 467-8601, Japan

E-mail: takeyama@med.nagoya-cu.ac.jp

Key words: laparoscopic surgery, carbon dioxide-pneumoperitoneum, sensory neurons, inflammatory responses, apoptosis

\section{Introduction}

Laparoscopic surgery provides multiple clinical benefits compared with open surgery, including decreased postoperative pain, shorter hospital stays, a more rapid return to preoperative activity, decreased postoperative ileus, preserved immune function and improved cosmetic results $(1,2)$. Furthermore, fewer inflammatory mediators are released following laparoscopic surgery than following conventional open surgery $(3,4)$. Abdominal laparoscopic surgery requires the intentional establishment of a pneumoperitoneum in order to provide adequate surgical exposure and operative freedom. Carbon dioxide $\left(\mathrm{CO}_{2}\right)$ is commonly used to induce the pneumoperitoneum as it is a colorless, stable gas that is, buffered in the blood, eliminated by the lung, is nonflammable and poses a low risk of venous gas embolism (5). $\mathrm{CO}_{2}$-pneumoperitoneum has been demonstrated to inhibit inflammatory cytokine release and neutrophil accumulation (6-8). However, the precise mechanisms underlying this effect are not fully understood.

Ablation of sensory neuronal fibers has been demonstrated to markedly increase inflammation severity suggesting that sensory neurons are important in maintaining tissue integrity by attenuating inflammatory responses (9). Capsaicin-sensitive sensory neurons are nociceptive neurons located around vessels and within the lining epithelia of numerous tissues, and are associated with nonvascular smooth muscle (10). Following stimulation of transient receptor potential vanilloid 1 (TRPV1) by a wide variety of noxious physical and chemical stimuli, including heat $\left(>42^{\circ} \mathrm{C}\right)$, low $\mathrm{pH}$, certain lipids and exogenous vanilloid derivatives, these sensory neurons release calcitonin gene-related peptide (CGRP) which is involved in the aggravation of inflammation such as tissue hyperemia and edema (9-11).

Our previous study demonstrated that stimulation of sensory neurons inhibits hepatic apoptosis and inflammatory responses in rats subjected to hepatic ischemia/reperfusion (I/R) (12). I/R-induced apoptosis increases neutrophil accumulation in damaged tissues (13) and activated neutrophils release various inflammatory mediators that are capable of damaging endothelial cells $(14,15)$. These observations suggest that excessive I/R-induced apoptosis permits a large number of neutrophils to accumulate at sites of damaged tissue 
and contribute to tissue injury by releasing inflammatory mediators that further damage endothelial and parenchymal cells. Consistent with this theory, our previous study demonstrated that reducing apoptosis in animal models of hepatic $\mathrm{I} / \mathrm{R}$ or water-immersion restraint stress inhibited neutrophil accumulation-reduced tissue injury (12).

Acidic environments (low $\mathrm{pH}$ ) are known to stimulate sensory neurons through activation of TRPV1 (16). Abdominal insufflation with $\mathrm{CO}_{2}$ causes a marked and rapid reduction in local tissue $\mathrm{pH}$ (17). In addition, $\mathrm{CO}_{2}$ has been demonstrated to stimulate sensory neurons through intracellular acidification by carbonic anhydrase $(18,19)$. These observations suggest that abdominal insufflation with $\mathrm{CO}_{2}$ may stimulate sensory neurons, thus inhibiting apoptosis and subsequent neutrophil accumulation.

Based on these observations, it was hypothesized that $\mathrm{CO}_{2}$-pneumoperitoneum may prevent I/R-induced inflammatory responses and hepatic apoptosis through stimulation of sensory neurons. In the present study, a rat model of I/R-induced liver injury was used to examine this hypothesis and to investigate the mechanism(s) by which laparoscopic surgery induces milder inflammatory responses than open surgery.

\section{Materials and methods}

Animals and reagents. Pathogen-free male Wistar rats, weighing 200-250 g, were obtained from Nihon SLC (Hamamatsu, Japan). Care and handling of the animals were conducted in accordance with the National Institutes of Health guidelines (Bethesda, MA, USA). All experimental procedures were approved by the Nagoya City University Animal Care Center (Nagoya, Aichi, Japan). SB366791, a specific TRPV1 antagonist (20), was purchased from Sigma-Aldrich (St. Louis, MO, USA). All reagents were of analytic grade.

Experimental model. The rats were randomly divided into the following five groups: sham-surgery, hepatic I/R, hepatic $\mathrm{I} / \mathrm{R}$ with $\mathrm{CO}_{2}$-pneumoperitoneum pretreatment, hepatic I/R with air-pneumoperitoneum pretreatment, hepatic $\mathrm{I} / \mathrm{R}$ with $\mathrm{CO}_{2}$-pneumoperitoneum and SB366791 pretreatment. All rats were deprived of food, but not of water, for $24 \mathrm{~h}$ prior to each experiment. The liver was exposed by a midline laparotomy after the induction of anesthesia. The ligation by silk was placed around the right and left branches of the portal vein, the hepatic artery and bile duct. The induction of ischemia of the median and left lobes of the liver was performed completely by clamping the left branches of the portal vein and hepatic artery for $60 \mathrm{~min}$. The right lobe of the liver was perfused to prevent the congestion of the intestine. The abdomen was covered with plastic wrap to prevent desiccation during the period of hepatic ischemia. During the period of hepatic ischemia, the abdomen was covered with plastic wrap to prevent desiccation. At the end of the period of ischemia, the ligatures around the left branches of the portal vein and hepatic artery were removed and the right branches of the portal vein, the hepatic artery and the bile duct were ligated to prevent a shunt-like effect to the right lobe following reperfusion $(21,22)$. This procedure directed all subsequent portal and hepatic blood flow, with the exception of a small amount of flow to the caudal hepatic lobe, through the previously ischemic liver lobes. The wound was closed with 3-0 silk. Sham-surgery animals were similarly handled, however, no ligature was placed to obstruct the blood flow to the left and median hepatic lobes. Blood flow to the right lobe of the liver was occluded in sham-surgery animals as in animals subjected to hepatic I/R. The rats assigned to the pneumoperitoneum group underwent either $\mathrm{CO}_{2}$ or air insufflation of their abdomen using a single 23-gauge needle for 30 min prior to hepatic ischemia. SB366791 was dissolved in normal saline with $1 \%$ dimethyl sulfoxide and $500 \mu \mathrm{g} / \mathrm{kg}$ was intraperitoneally administered $60 \mathrm{~min}$ prior to ischemia as described previously (23). The median lobe of the liver was removed following the indicated period of reperfusion for histological analysis as described below.

Histology and immunohistochemistry. Following $6 \mathrm{~h}$ of hepatic $\mathrm{I} / \mathrm{R}$, the rats were perfused with $4 \%$ paraformaldehyde in $0.1 \mathrm{M}$ phosphate-buffered saline. The median lobe of the liver was removed and embedded in paraffin. Paraffin-embedded samples were sectioned every $3 \mu \mathrm{m}$ and then deparaffinized. Apoptosis was assessed with hematoxylin and eosin (H\&E) and the terminal deoxynucleotidyl transferase dUTP nick end-labeling (TUNEL) staining methods. In H\&E stained sections, cells with morphological features of apoptosis, including cell shrinkage, retraction of cell borders and chromatin condensation and margination were counted $(24,25)$. TUNEL staining was performed with the MEBSTAIN Apoptosis kit direct (MBL Co., Nagoya, Japan) as described previously $(26,27)$. The number of apoptotic cells within each of five randomly selected high-power microscopy fields (HPF) at X400 magnification (BZ9000; Keyence, Osaka, Japan) was determined and the data are expressed as the average number of TUNEL-positive cells per HPF.

Immunofluorescence staining of endothelial monocyte-activated polypeptide-II (EMAP-II) was performed by pretreating the sections with proteinase $\mathrm{K}$ (1:40 dilution) and then blocking with Tris/NaCl/blocking reagent buffer (TNB; Perkin Elmer Life Sciences, Boston, MA, USA) for $1 \mathrm{~h}$ at room temperature. The sections were then incubated with the primary antibody (monoclonal mouse anti-EMAP-II; Abcam, Cambridge, UK) diluted 1:100 in TNB overnight at $4^{\circ} \mathrm{C}$, and then washed and incubated with Alexa Fluorophore $568 \mathrm{~nm}$ polyclonal donkey anti-mouse antibody (Invitrogen, Mount Waverley, Australia) at 1:500 dilution in TNB for $1 \mathrm{~h}$ at room temperature. The sections were counterstained with 4'-6-diamidino-2-phenylindole (Sigma-Aldrich). In the control experiments, primary antibodies were omitted to verify the absence of non-specific binding of secondary antibodies. Staining intensity was semi-quantitatively scored as 0 (negative), 1 (weak), 2 (moderate) and 3 (strong), and the proportion of cells staining positively at any intensity was scored as 0 ( $<5 \%$ of cells), 1 (5-30\% of cells), 2 (31-60\% of cells) and 3 ( $>60 \%$ of cells), as described previously $(28,29)$. The intensity and proportion scores were added for each sample to give the immunohistochemistry scores, which ranged between 0 and 6 .

Statistical analysis. Data are expressed as the mean \pm standard deviation. The results were compared by analysis of variance followed by Bonferroni's multiple comparison test. $\mathrm{P}<0.05$ was considered to indicate a statistically significant difference. 
A

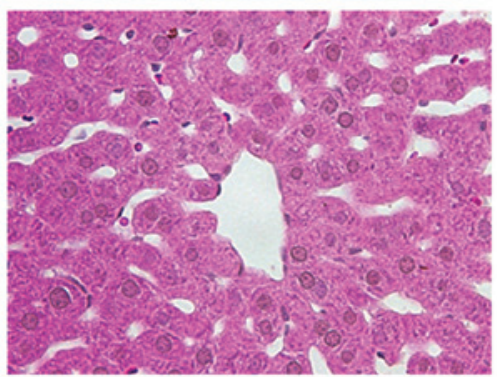

D

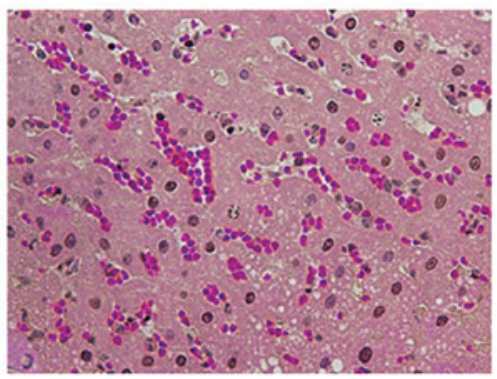

B

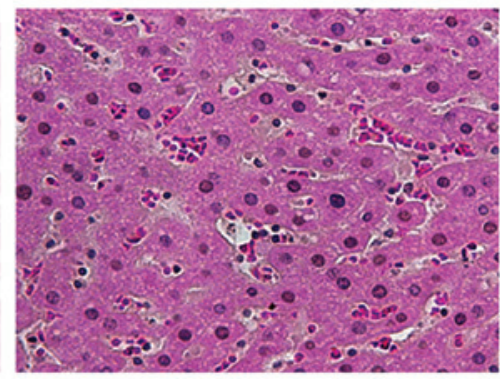

E

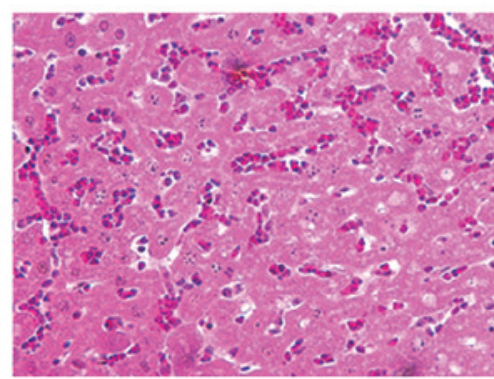

C

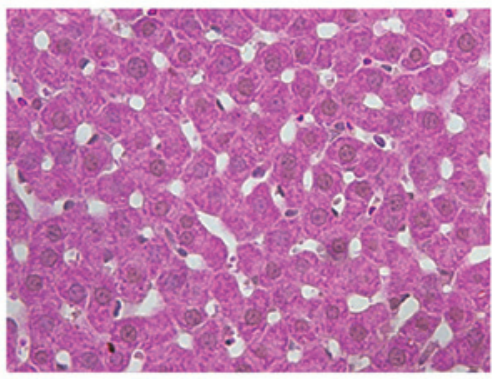

F

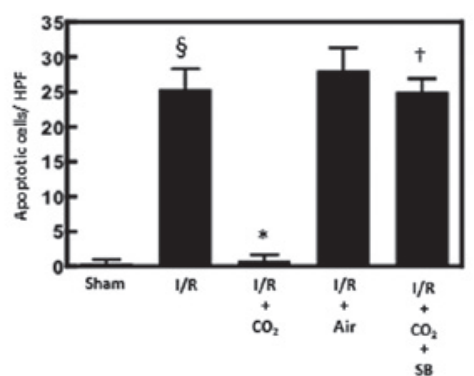

Figure 1. Effects of $\mathrm{CO}_{2}$-pneumoperitoneum, air-pneumoperitoneum and SB366791 on H\&E staining $6 \mathrm{~h}$ after hepatic I/R. H\&E staining was performed $6 \mathrm{~h}$ after reperfusion in rats subjected to hepatic I/R (original magnification, x400). (A) Sham; (B) I/R; (C) I/R + CO ; (D) I/R + air; (E) I/R + CO $+\mathrm{SB}$. Typical results from five separate experiments are shown. (F) Comparison of the number of cells with morphological features of apoptosis in the liver following hepatic I/R. Apoptotic cells were counted in five HPFs of a light microscope ( $\mathrm{n}=5$ for each group). Each bar represents the mean \pm standard deviation. ${ }^{\S} \mathrm{P}<0.01$ vs. sham; ${ }^{*} \mathrm{P}<0.01$ vs. I/R; ${ }^{\dagger} \mathrm{P}<0.01$ vs. I/R $+\mathrm{CO}_{2}$. H\&E, hematoxylin and eosin; $\mathrm{CO}_{2}$, carbon dioxide; HPF, high-power microscopy field; I/R, ischemia/reperfusion; SB, SB366791.

\section{Results}

Effects of $\mathrm{CO}_{2}$ - and air-pneumoperitoneum on hepatic apoptosis in rats subjected to hepatic I/R. Hepatic apoptosis has been demonstrated to be critical in the development of I/R-induced tissue injury by inducing inflammatory responses (13). The effects of $\mathrm{CO}_{2}$ - and air-pneumoperitoneum on the number of apoptotic cells in the livers of rats subjected to hepatic I/R were examined using H\&E and TUNEL staining methods. Compared with sham surgery, hepatic I/R increased the number of apoptotic cells observed following $6 \mathrm{~h}$ of reperfusion $(\mathrm{P}<0.01)$. $\mathrm{CO}_{2}$-pneumoperitoneum inhibited this increase $(\mathrm{P}<0.01)$, however, air-pneumoperitoneum did not (Figs. 1 and 2).

Effects of $\mathrm{CO}_{2}$ - and air-pneumoperitoneum on hepatic EMAP-II expression in rats subjected to hepatic I/R. EMAP-II is a chemoattractant for monocytes and neutrophils (30) and is produced by apoptotic cells by cleavage of the precursor protein proEMAP-II (31). It was postulated that $\mathrm{CO}_{2}$-pneumoperitoneum inhibited I/R-induced hepatic apoptosis by inhibiting the hepatic activation of EMAP-II during I/R. To examine this possibility, the effects of $\mathrm{CO}_{2}$ - and air-pneumoperitoneum on EMAP-II expression were analyzed in the livers of rats subjected to hepatic I/R. Immunofluorescence staining for EMAP-II in the livers of animals subjected to hepatic I/R was increased $6 \mathrm{~h}$ after reperfusion compared with the sham-surgery animals $(\mathrm{P}<0.01$; Fig. 3). $\mathrm{CO}_{2}$-pneumoperitoneum reduced this increase more strongly compared with that of air pneumoperitoneum (Fig. 3).

Effect of SB366791 on alterations induced by $\mathrm{CO}_{2}$-pneumoperitoneum in rats subjected to hepatic I/R. To determine whether $\mathrm{CO}_{2}$-pneumoperitoneum reduces
I/R-induced hepatic apoptosis and inflammatory responses by activating TRPV1 in sensory neurons, the effects of the specific TRPV1 antagonist SB366791 on alterations induced by $\mathrm{CO}_{2}$-pneumoperitoneum were analyzed in rats subjected to hepatic I/R. Administration of SB366791 completely reversed the ability of $\mathrm{CO}_{2}$-pneumoperitoneum to inhibit I/R-induced apoptosis and hepatic EMAP-II expression $(\mathrm{P}<0.05$; Figs. 1-3).

\section{Discussion}

The present study demonstrated that $\mathrm{CO}_{2}$-pneumoperitoneum prevented I/R-induced hepatic apoptosis, while air-pneumoperitoneum did not. Pretreatment with the specific TRPV1 antagonist SB366791 abrogates this effect. Our previous study demonstrated that stimulation of sensory neurons in animals subjected to hepatic I/R inhibits hepatic apoptosis by inducing CGRP (12). These observations suggest that $\mathrm{CO}_{2}$-pneumoperitoneum stimulates sensory neurons through TRPV1 activation, thereby inhibiting hepatic apoptosis in rats following hepatic I/R. Since hepatic apoptosis was not inhibited by air-pneumoperitoneum, it is likely that abdominal distension alone does not induce this effect.

The precise mechanisms by which $\mathrm{CO}_{2}$-pneumoperitoneum activates sensory neurons are not clear. Acidic environments (low $\mathrm{pH}$ ) are known to stimulate sensory neurons through activation of TRPV1 (16), and abdominal insufflation with $\mathrm{CO}_{2}$ causes a marked and rapid reduction in local tissue $\mathrm{pH}$ (17). Furthermore, $\mathrm{CO}_{2}$ has been demonstrated to stimulate sensory neurons through intracellular acidification by carbonic anhydrase $(18,19)$ and to stimulate CGRP release through a decrease in intracellular $\mathrm{pH}$ in sensory neurons in vitro (32). 
A

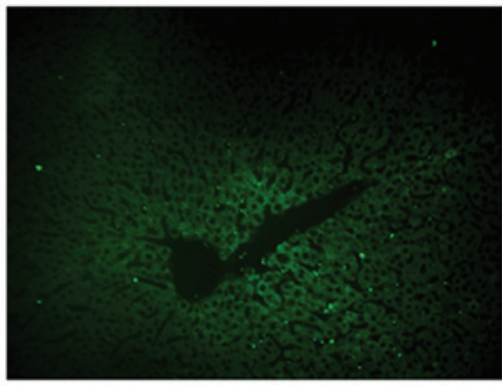

D

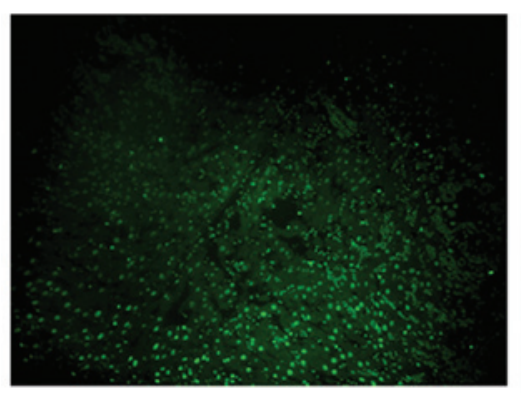

B

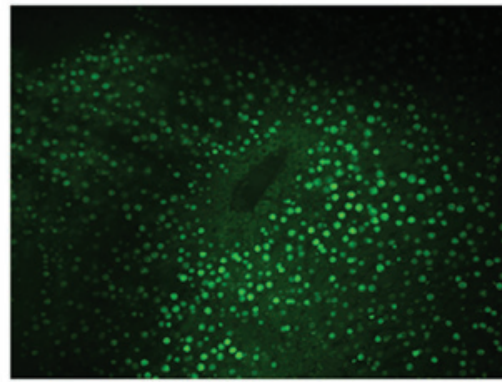

E

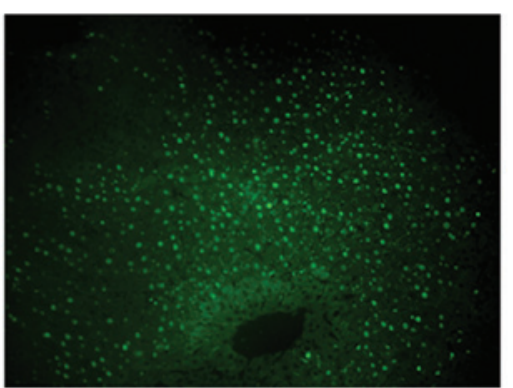

C

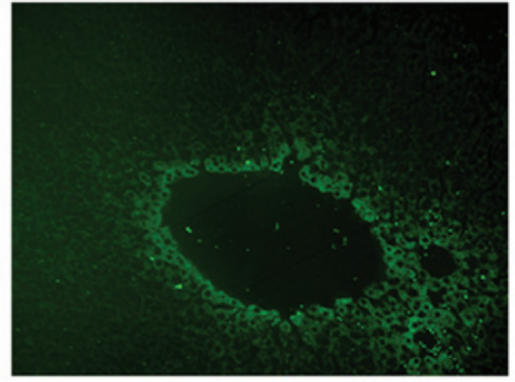

F

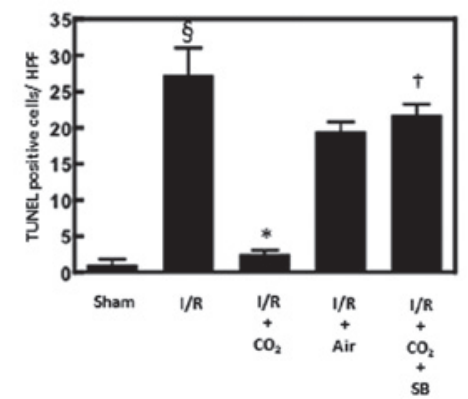

Figure 2. Effects of $\mathrm{CO}_{2}$-pneumoperitoneum, air-pneumoperitoneum and SB366791 on TUNEL staining $6 \mathrm{~h}$ after hepatic $\mathrm{I} / \mathrm{R}$ in rats. TUNEL staining was performed $6 \mathrm{~h}$ after reperfusion in rats subjected to hepatic I/R (original magnification, $\mathrm{x} 200$ ). (A) Sham; (B) $\mathrm{I} / \mathrm{R} ;(\mathrm{C}) \mathrm{I} / \mathrm{R}+\mathrm{CO}_{2}$; (D) $\mathrm{I} / \mathrm{R}+$ air; (E) $\mathrm{I} / \mathrm{R}+\mathrm{CO}+\mathrm{SB}$. Typical results from four separate experiments are shown. (F) Comparison of the number of TUNEL-positive cells in the liver following hepatic I/R. TUNEL-positive cells were counted in four HPFs of an immunofluorescent microscope ( $\mathrm{n}=4$ for each group). Each bar represents the mean \pm standard deviation. ${ }^{\circledR} \mathrm{P}<0.01 \mathrm{vs}$. sham; "P $<0.01$ vs. I/R; ${ }^{\dagger} \mathrm{P}<0.01$ vs. I/R $+\mathrm{CO}_{2}$. $\mathrm{CO}_{2}$, carbon dioxide; TUNEL, terminal deoxynucleotidyl transferase dUTP nick end-labeling; I/R, ischemia/reperfusion; HPF, high-power microscopy field; SB, SB366791.

A

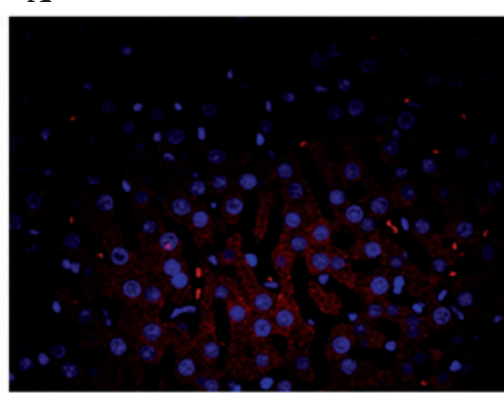

D

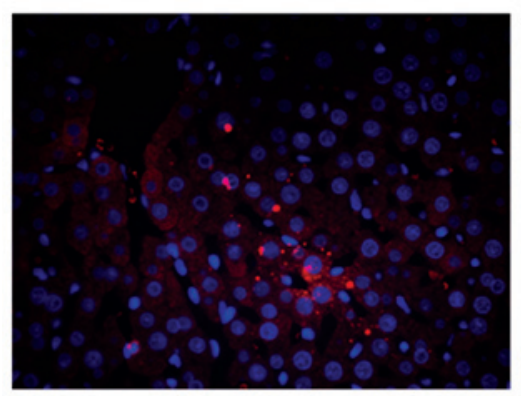

B

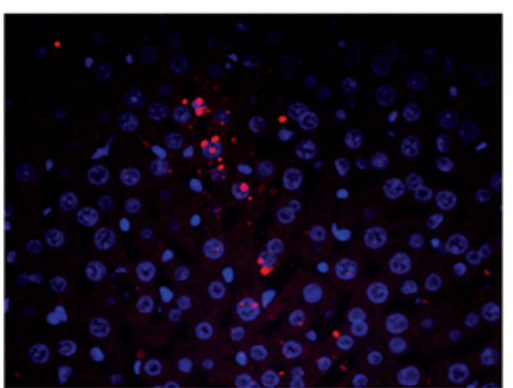

$\mathbf{E}$

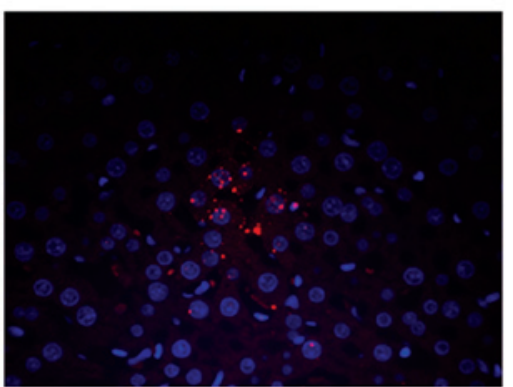

C

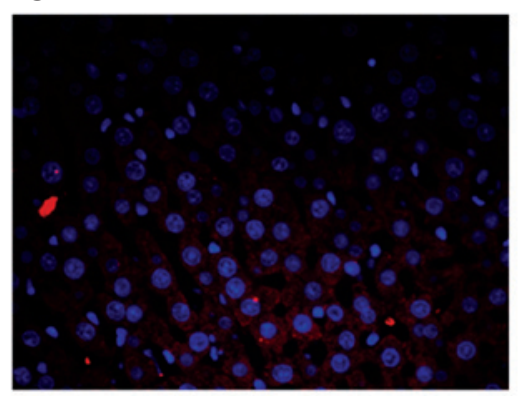

F

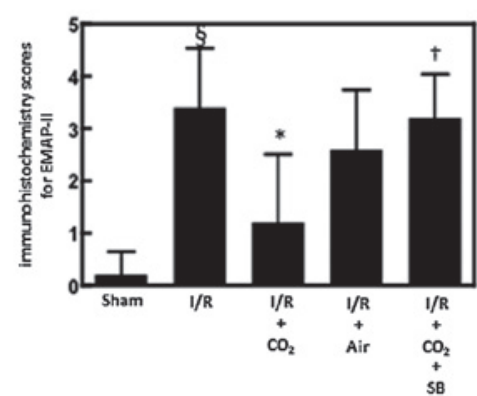

Figure 3. Effects of $\mathrm{CO}_{2}$-pneumoperitoneum, air-pneumoperitoneum and SB366791 on hepatic EMAP-II expression $6 \mathrm{~h}$ after hepatic I/R in rats. Immunofluorescent EMAP-II staining (red) of liver tissue was performed $6 \mathrm{~h}$ after reperfusion in rats subjected to hepatic I/R (original magnification, $\mathrm{x} 400$ ). Nuclei were counterstained with 4'-6-diamidino-2-phenylindole (blue). (A) Sham; (B) I/R; (C) I/R + $\mathrm{CO}_{2}$; (D) I/R + air; (E) I/R + CO + SB. Typical results from five separate experiments are shown. (F) Comparison of the immunohistochemistry scores for EMAP-II staining of the liver following hepatic I/R (n=5 for each group). Each bar represents the mean \pm standard deviation. ${ }^{8} \mathrm{P}<0.01$ vs. sham; ${ }^{*} \mathrm{P}<0.01 \mathrm{vs.} \mathrm{I} / \mathrm{R}$; ${ }^{\dagger} \mathrm{P}<0.05 \mathrm{vs}$. I/R $+\mathrm{CO}_{2}$. $\mathrm{CO}_{2}$, carbon dioxide; EMAP-II, endothelial monocyte-activated polypeptide-II; I/R, ischemia/reperfusion; SB, SB366791.

These observations suggest that abdominal insufflation with $\mathrm{CO}_{2}$ may stimulate sensory neurons by decreasing intracellular $\mathrm{pH}$.
$\mathrm{CO}_{2}$-pneumoperitoneum strongly inhibited I/R-induced increases in the hepatic expression of EMAP-II compared with that of air-pneumoperitoneum. Pretreatment with SB366791 
completely reversed this effect. These observations suggest that $\mathrm{CO}_{2}$-pneumoperitoneum, and not abdominal distension alone, may inhibit I/R-induced increases in EMAP-II expression by activating TRPV1. EMAP-II is a chemoattractant for neutrophils (30). Its precursor protein, proEMAP-II, is cleaved by caspase- 3 to the biologically active form (33). Caspases are activated in apoptotic cells. Following renal I/R, caspase activation increases neutrophil accumulation in the kidney by increasing EMAP-II expression (13). These observations suggest that, in addition to inducing apoptosis, caspases contribute to tissue inflammation and injury by increasing neutrophil infiltration at damaged sites through activation of EMAP-II. Our previous study demonstrated that stimulation of sensory neurons inhibits I/R-induced increases of caspase-3 and apoptosis in hepatic tissue (12). Thus, it is possible that $\mathrm{CO}_{2}$-pneumoperitoneum prior to hepatic I/R inhibits caspase activation through stimulation of sensory neurons, thereby attenuating hepatic apoptosis and the expression of EMAP-II.

Based on our results, it was postulated that $\mathrm{CO}_{2}$-pneumoperitoneum stimulates sensory neurons in the liver, thereby inhibiting I/R-induced inflammatory responses and hepatic apoptosis. Our results further suggest that the effects of $\mathrm{CO}_{2}$-pneumoperitoneum are unlikely to be associated with abdominal distension alone. Inflammatory responses are critical in the development of post-operative complications (34), and the present study indicates that $\mathrm{CO}_{2}$-pneumoperitoneum may contribute to the clinical benefits of laparoscopic surgery by attenuating inflammatory responses via stimulation of sensory neurons.

Ischemic preconditioning, the most effective gastroprotective intervention, involves sensory neurons $(35,36)$. The mechanism underlying the beneficial effects of laparoscopic surgery may be similar to those involved in ischemic preconditioning. It is thus intriguing to consider the possibility that $\mathrm{CO}_{2}$-pneumoperitoneum may be beneficial when performed as a preconditioning stimulus, that is prior to open surgery. This possibility should be examined in the clinical setting.

\section{References}

1. Mealy K, Gallagher H, Barry M, Lennon F, Traynor O and Hyland J: Physiological and metabolic responses to open and laparoscopic cholecystectomy. Br J Surg 79: 1061-1064, 1992.

2. Zmora O, Hashavia E, Munz Y, Khaikin M, Shabtai M, Ayalon A, Dinur L and Rosin D: Laparoscopic colectomy is associated with decreased postoperative gastrointestinal dysfunction. Surg Endosc 23: 87-89, 2009.

3. Redmond HP, Watson RW, Houghton T, Condron C, Watson RG and Bouchier-Hayes D: Immune function in patients undergoing open vs laparoscopic cholecystectomy. Arch Surg 129: 1240-1246, 1994.

4. Vittimberga FJ Jr, Foley DP, Meyers WC and Callery MP: Laparoscopic surgery and the systemic immune response. Ann Surg 227: 326-334, 1998

5. Mann C, Boccara G, Grevy V, Navarro F, Fabre JM and Colson P: Argon pneumoperitoneum is more dangerous than $\mathrm{CO}_{2}$ pneumoperitoneum during venous gas embolism. Anesth Analg 85: 1367-1371, 1997.

6. Araújo Filho I, Honorato Sobrinho AA, Rego AC, Garcia AC, Fernandes DP, Cruz TM, Costa TC and Medeiros AC: Influence of laparoscopy and laparotomy on gasometry, leukocytes and cytokines in a rat abdominal sepsis model. Acta Cir Bras 21: 74-79, 2006.

7. Hanly EJ, Fuentes JM, Aurora AR, Bachman SL, De Maio A, Marohn MR and Talamini MA: Carbon dioxide pneumoperitoneum prevents mortality from sepsis. Surg Endosc 20: 1482-1487, 2006.
8. Pitombo MB, Lupi OH, Gomes RN, Amâncio R, Refinetti RA, Bozza PT and Castro-Faria-Neto HC: Inflammatory response and bacterial dissemination after laparotomy and abdominal $\mathrm{CO}_{2}$ insufflation in a murine model of peritonitis. Surg Endosc 20: 1440-1447, 2006.

9. Okajima K and Harada N: Regulation of inflammatory responses by sensory neurons: molecular mechanism(s) and possible therapeutic applications. Curr Med Chem 13: 2241-2251, 2006.

10. Maggi CA and Meli A: The sensory-efferent function of capsaicin-sensitive sensory neurons. Gen Pharmacol 19: 1-43, 1988.

11. Caterina MJ, Schumacher MA, Tominaga M, Rosen TA, Levine JD and Julius D: The capsaicin receptor: a heat-activated ion channel in the pain pathway. Nature 389: 816-824, 1997.

12. Kawai M, Harada N, Takeyama H and Okajima K: Neutrophil elastase contributes to the development of ischemia/reperfusion-induced liver injury by decreasing the production of insulin-like growth factor-I in rats. Transl Res 155: 294-304, 2010.

13. Daemen MA, van 't Veer C, Denecker G, Heemskerk VH, Wolfs TG, Clauss M, Vandenabeele P and Buurman WA: Inhibition of apoptosis induced by ischemia-reperfusion prevents inflammation. J Clin Invest 104: 541-549, 1999.

14. Jaeschke H: Molecular mechanisms of hepatic ischemia-reperfusion injury and preconditioning. Am J Physiol Gastrointest Liver Physiol 284: G15-G26, 2003.

15. Massip-Salcedo M, Roselló-Catafau J, Prieto J, Avila MA and Peralta C: The response of the hepatocyte to ischemia. Liver Int 27: 6-16, 2007.

16. Holzer P: The pharmacological challenge to tame the transient receptor potential vanilloid-1 (TRPV1) nocisensor. $\mathrm{Br}$ J Pharmacol 155: 1145-1162, 2008.

17. West MA, Hackam DJ, Baker J, Rodriguez JL, Bellingham J and Rotstein OD: Mechanism of decreased in vitro murine macrophage cytokine release after exposure to carbon dioxide: relevance to laparoscopic surgery. Ann Surg 226: 179-190, 1997.

18. Akiba Y, Ghayouri S, Takeuchi T, Mizumori M, Guth PH, Engel E, Swenson ER and Kaunitz JD: Carbonic anhydrases and mucosal vanilloid receptors help mediate the hyperemic response to luminal $\mathrm{CO}_{2}$ in rat duodenum. Gastroenterology 131: 142-152, 2006.

19. Prabhakar E and Lawson SN: The electrophysiological properties of rat primary afferent neurones with carbonic anhydrase activity. J Physiol 482: 609-622, 1995.

20. Varga A, Németh J, Szabó A, McDougall JJ, Zhang C, Elekes K, Pintér E, Szolcsányi J and Helyes Z: Effects of the novel TRPV1 receptor antagonist SB366791 in vitro and in vivo in the rat. Neurosci Lett 385: 137-142, 2005.

21. Hayashi H, Chaudry IH, Clemens MG and Baue AE: Hepatic ischemia models for determining the effects of ATP- $\mathrm{MgCl}_{2}$ treatment. J Surg Res 40: 167-175, 1986.

22. Jaeschke H, Farhood A, Bautista AP, Spolarics Z, Spitzer JJ and Smith CW: Functional inactivation of neutrophils with a Mac-1 (CD11b/CD18) monoclonal antibody protects against ischemia-reperfusion injury in rat liver. Hepatology 17: 915-923, 1993.

23. Nakayama T, Harada N, Asano M, Nomura N, Saito T, Mishima A and Okajima K: Atrial natriuretic peptide reduces ischemia/reperfusion-induced spinal cord injury in rats by enhancing sensory neuron activation. J Pharmacol Exp Ther 322: 582-590, 2007.

24. Soeda J, Miyagawa S, Sano K, Masumoto J, Taniguchi S and Kawasaki S: Cytochrome c release into cytosol with subsequent caspase activation during warm ischemia in rat liver. Am J Physiol Gastrointest Liver Physiol 281: G1115-G1123, 2001.

25. Martin EJ and Forkert PG: Evidence that 1,1-dichloroethylene induces apoptotic cell death in murine liver. J Pharmacol Exp Ther 310: 33-42, 2004

26. Kelly KJ, Sandoval RM, Dunn KW, Molitoris BA and Dagher PC: A novel method to determine specificity and sensitivity of the TUNEL reaction in the quantitation of apoptosis. Am J Physiol Cell Physiol 284: C1309-C1318, 2003.

27. Gavrieli Y, Sherman Y and Ben-Sasson SA: Identification of programmed cell death in situ via specific labeling of nuclear DNA fragmentation. J Cell Biol 119: 493-501, 1992.

28. Chaerkady R, Harsha HC, Nalli A, Gucek M, Vivekanandan P, Akhtar J, Cole RN, Simmers J, Schulick RD, Singh S, Torbenson M, Pandey A and Thuluvath PJ: A quantitative proteomic approach for identification of potential biomarkers in hepatocellular carcinoma. J Proteome Res 7: 4289-4298, 2008. 
29. Barnes DM, Harris WH, Smith P, Millis RR and Rubens RD: Immunohistochemical determination of oestrogen receptor: comparison of different methods of assessment of staining and correlation with clinical outcome of breast cancer patients. $\mathrm{Br}$ J Cancer 74: 1445-1451, 1996.

30. Kao J, Fan YG, Haehnel I, Brett J, Greenberg S, Clauss M, Kayton M, Houck K, Kisiel W, Seljelid R, et al: A peptide derived from the amino terminus of endothelial-monocyte-activating polypeptide II modulates mononuclear and polymorphonuclear leukocyte functions, defines an apparently novel cellular interaction site, and induces an acute inflammatory response. J Biol Chem 269: 9774-9782, 1994

31. Knies UE, Behrensdorf HA, Mitchell CA, Deutsch U, Risau W, Drexler HC and Clauss M: Regulation of endothelial monocyte-activating polypeptide II release by apoptosis. Proc Natl Acad Sci USA 95: 12322-12327, 1998.

32. Vause C, Bowen E, Spierings E and Durham P: Effect of carbon dioxide on calcitonin gene-related peptide secretion from trigeminal neurons. Headache 47: 1385-1397, 2007.
33. van Horssen R, Eggermont AM and ten Hagen TL: Endothelial monocyte-activating polypeptide-II and its functions in (patho) physiological processes. Cytokine Growth Factor Rev 17: 339-348, 2006.

34. Jacobi CA, Wenger F, Opitz I and Müller JM: Immunologic changes during minimally invasive surgery. Dig Surg 19: 459-463, 2002.

35. Pawlik M, Ptak A, Pajdo R, Konturek PC, Brzozowski T and Konturek SJ: Sensory nerves and calcitonin gene related peptide in the effect of ischemic preconditioning on acute and chronic gastric lesions induced by ischemia-reperfusion. J Physiol Pharmacol 52: 569-581, 2001.

36. Pajdo R, Brzozowski T, Konturek PC, Kwiecien S, Konturek SJ, Sliwowski Z, Pawlik M, Ptak A, Drozdowicz D and Hahn EG: Ischemic preconditioning, the most effective gastroprotective intervention: involvement of prostaglandins, nitric oxide, adenosine and sensory nerves. Eur J Pharmacol 427: 263-276, 2001. 